Article

\title{
Antimicrobial Efficacy of Five Probiotic Strains Against Helicobacter pylori
}

\author{
Ilaria Maria Saracino ${ }^{1,+}+$, Matteo Pavoni ${ }^{1,+}+{ }^{\circ}$, Laura Saccomanno ${ }^{1}$, Giulia Fiorini ${ }^{1}$, \\ Valeria Pesci ${ }^{1}$, Claudio Foschi ${ }^{2}{ }^{\oplus}$, Giulia Piccirilli ${ }^{2}$, Giulia Bernardini ${ }^{3}{ }^{(}$, John Holton ${ }^{4}$, \\ Natale Figura ${ }^{3}$, Tiziana Lazzarotto ${ }^{2}$, Claudio Borghi ${ }^{1}$ and Berardino Vaira ${ }^{1, *}$ \\ 1 Department of Surgical and Medical Sciences, University of Bologna, 40138 Bologna, Italy; \\ saracinoilariamaria@gmail.com (I.M.S.); matteo.pavoni@studio.unibo.it (M.P.); \\ laura.saccomanno@studio.unibo.it (L.S.); giulia.fiorini@fastwebnet.it (G.F.); valeria.pesci@gmail.com (V.P.); \\ claudio.borghi@unibo.it (C.B.) \\ 2 Microbiology and Clinical Microbiology, Department of Experimental, Diagnostic and Specialty Medicine, \\ University of Bologna, 40138 Bologna, Italy; claudio.foschi2@unibo.it (C.F.); giulia.piccirilli2@unibo.it (G.P.); \\ tiziana.lazzarotto@unibo.it (T.L.) \\ 3 Department of Biotechnology Chemistry and Pharmacy, University of Siena, 53100 Siena, Italy; \\ bernardini@unisi.it (G.B.); figuranatale@gmail.com (N.F.) \\ 4 Department of Health \& Social Sciences, University of Middlesex, London NW4 4HE, UK; \\ john.holton@nhs.net \\ * Correspondence: berardino.vaira@unibo.it; Tel./Fax: +39-0512-144-140 \\ + These authors contributed equally to this work.
}

Received: 3 April 2020; Accepted: 9 May 2020; Published: 11 May 2020

check for updates

\begin{abstract}
Treatment of Helicobacter pylori (H. pylori) infection is a challenge for clinicians. The large increase in drug-resistant strains makes the formulation of new therapeutic strategies fundamental. The frequent onset of side effects during antibiotic treatment (mainly due to intestinal dysbiosis) should not be underestimated as it may cause the interruption of treatment, failure of H. pylori eradication and clonal selection of resistant bacteria. Probiotic integration during antibiotic treatment can exert a dual function: a direct antagonistic effect on $\mathrm{H}$. pylori and a balancing effect on dysbiosis. Therefore, it fulfills the definition of a new therapeutic strategy to successfully treat $H$. pylori infection. Data reported in literature give promising but discrepant results. Aim: To assess in vitro bacteriostatic and bactericidal activity of probiotic strains against $H$. pylori. Materials and methods: L. casei, $L$. paracasei, L. acidophilus, B. lactis and S. thermophilus strains were used. Agar well diffusion and time-kill curves were carried out to detect bacteriostatic and bactericidal activity, respectively. Results: All probiotic strains showed both bacteriostatic and bactericidal activity vs. H. pylori. Conclusions: Such findings prompted us to plan a protocol of treatment in which probiotics are given to infected patients in association with antibiotic therapy.
\end{abstract}

Keywords: probiotics; Helicobacter pylori; dysbiosis; therapy; bactericidal activity; bacteriostatic activity

\section{Introduction}

Helicobacter pylori is a Gram-negative microaerophilic bacterium that colonizes the gastric mucosa, causing gastritis and peptic ulcers. It leads to the development of gastric-mucosa-associated lymphoid tissue (MALT) lymphoma and gastric carcinoma [1]. Treatment of $H$. pylori infection is a challenge for clinicians and the large increase in drug-resistant strains globally makes the development of new therapeutic approaches crucial [2]. Additionally, not to be underestimated is the clinical significance of frequent adverse events caused by current antibiotic treatments (mainly due to intestinal dysbiosis). 
These adverse events result in interruption of therapy, favoring the clonal selection of resistant strains and creating a vicious cycle [3].

Several bacterial Phyla have been identified in the stomach. The gastric microbiota is dynamic and is affected by several factors, establishing multiple interactions with the gastric mucosa and, when present, with $H$. pylori. Overall, in the presence of $H$. pylori, a loss of microbiota biodiversity occurs, leading to dysbiosis. Probiotic use for an $\mathrm{H}$. pylori infected stomach has many beneficial immunological and non-immunological effects: it enables the rebalancing of pro- and anti-inflammatory cytokines, activates defense mechanisms against pathogens and improves the mucosal barrier. Furthermore, probiotics compete with $\mathrm{H}$. pylori for the same adhesion sites and nutrients and induce the production of mucin; they also produce metabolites with antimicrobial activity $[4,5]$. Probiotic integration during antibiotic therapies can also balance intestinal dysbiosis, thus decreasing dysbiosis-induced adverse events and increasing patient compliance. For these reasons, integration with probiotics fully responds to the need of new therapeutic cues to successfully treat $H$. pylori infection. A recent meta-analysis stated that "probiotics improved the eradication rate and reduced side effects when added to the treatments designed to eradicate $H$ pylori. The use of probiotics either before and throughout the eradication treatment, exerted better eradication effects" [6]. However, data reported in the literature give discrepant results. The aim of this study was to assess in vitro the bacteriostatic and bactericidal activity of probiotic strains against $H$. pylori, as a preliminary experiment to plan a clinical trial.

\section{Results}

Viable counts (range, mean, standard deviation) and $\mathrm{pH}$ ranges of probiotic strains after overnight culture are reported in Table 1 . It was not possible to normalize the quantity of cells after overnight cultures due to the different bacterial growth rates. Overnight broth-cultures were not diluted in order not to modify their metabolite content.

Table 1. Cells/mL and $\mathrm{pH}$ range after 20-h overnight culture.

\begin{tabular}{ccccc}
\hline Probiotic Strain & $\mathbf{N}^{\circ}$ of Cells/mL (Range) & Means & SD & pH Range \\
\hline L. casei & $9 \times 10^{9}-1 \times 10^{10}$ & $9.4 \times 10^{9}$ & $3.1 \times 10^{8}$ & 4 to 4.5 \\
L. paracasei & $6.3 \times 10^{9}-1 \times 10^{10}$ & $7.7 \times 10^{9}$ & $1 \times 10^{9}$ & 4 to 4.5 \\
L. acidophilus & $6.6 \times 10^{9}-8 \times 10^{9}$ & $7.1 \times 10^{9}$ & $4.5 \times 10^{8}$ & 4 to 4.5 \\
B. lactis & $3.6 \times 10^{9}-7.5 \times 10^{9}$ & $6.1 \times 10^{9}$ & $1.8 \times 10^{9}$ & 4 to 4.5 \\
S. thermophilus & $3 \times 10^{9}-6.9 \times 10^{9}$ & $3.7 \times 10^{9}$ & $1.4 \times 10^{9}$ & 5 to 6 \\
\hline
\end{tabular}

SD: standard deviation.

Fifty-seven H. pylori strains, grouped according to the eight resistance patterns observed, were tested (Table 2). Antibiotic susceptibility tests were performed with the E-test method.

Table 2. H. pylori strains tested with agar well diffusion.

\begin{tabular}{cc}
\hline Resistance Patterns & Number of Strains \\
\hline ClaS, MzS, LS & 9 \\
ClaR, MzR, LR & 10 \\
ClaR, MzS, LS & 9 \\
ClaR, MzR, LS & 10 \\
ClaS, MzR, LS & 4 \\
ClaS, MzS, LR & 3 \\
ClaS, MzR, LR & 5 \\
ClaR, MzS, LR & 7 \\
\hline Total & 57 \\
\hline
\end{tabular}

Cla: clarithromycin. Mz: metronidazole. L: levofloxacin. S: susceptible. R: resistant.

All probiotic strains showed bacteriostatic activity against H. pylori (Figure 1). All the five strains generated inhibition zones (IZs) larger than those of the respective negative controls (MRS (DeMan-Rogosa-Sharpe) and BHI (brain heart infusion) broths) $(p<0.05)$. IZs obtained with L. casei, L. 
paracasei and L. acidophilus were very similar to each other and were greater than those generated by $B$. lactis, which in turn created larger IZs than those generated by $S$. thermophilus. These differences were statistically significant $(p<0.05)$. IZ means and their standard errors are reported in Figure 1.

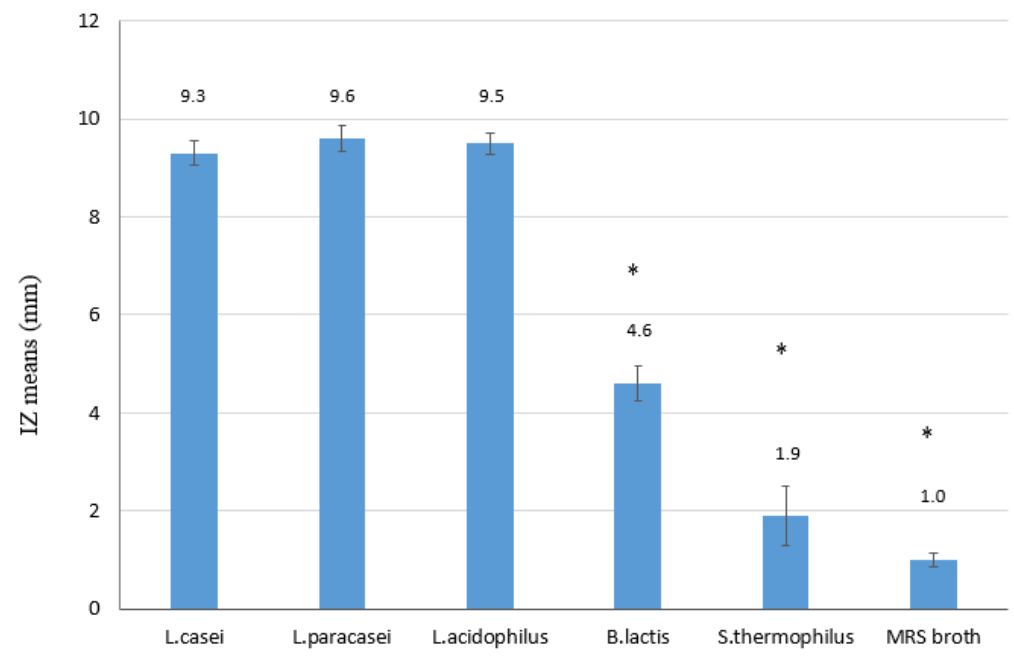

Figure 1. Inhibition zone (IZ) means of the five probiotic strains against H. pylori. DeMan-Rogosa-Sharpe (MRS) and Brain Heart Infusion (BHI) broths were used as negative controls. BHI broth never produced an IZ. IZs trend: L. casei $=$ L. paracasei $=$ L. acidophilus $>$ B. lactis $>$ S. thermophiles $>$ negative control. ${ }^{*} p<0.05$ was considered significant.

No differences were observed in the IZ means of the same probiotic strain vs. the eight $H$. pylori groups. This confirms that H. pylori antibiotic resistance mechanisms do not interfere with its susceptibility to the antimicrobial activity of probiotic strains (Figure 2).

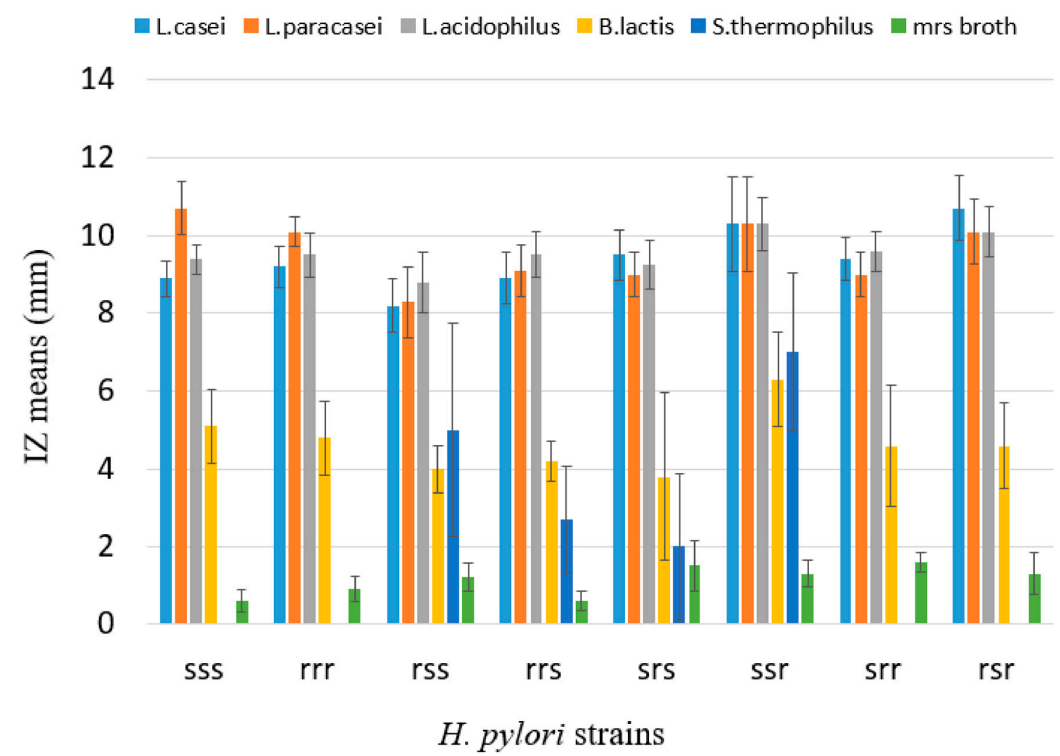

Figure 2. IZ means (and standard errors) in the eight H. pylori groups with different resistance patterns. S: susceptible. R: resistant. Resistance patterns are reported in this order: clarithromycin, metronidazole, levofloxacin. BHI broth never produced an IZ. IZs do not correlate with H. pylori pattern of antibiotic resistance. IZ distributions and means of the same probiotic strain vs. the eight different $H$. pylori groups were analyzed using ANOVA and Student's $t$-test; no statistically significant difference was detected. Differences of IZ means were still significant between the probiotic strains as previously reported: $L$. casei $=$ L. paracasei $=$ L. acidophilus $>$ B. lactis $>$ S. thermophiles $>$ negative cntr. 
L. casei, L. paracasei and L. acidophilus inhibited all H. pylori strains; B. lactis inhibited 89.5\% (51/57) of them; S. thermophilus inhibited 18\% (10/57) of them but its IZs, when present, were large.

IZ range for L. casei, L. paracasei, L. acidophilus, B. lactis and S. thermophilus were, respectively, $4 \mathrm{~mm}$ to $14 \mathrm{~mm}, 4 \mathrm{~mm}$ to $15 \mathrm{~mm}, 4 \mathrm{~mm}$ to $14 \mathrm{~mm}$, no inhibition to $10 \mathrm{~mm}$ and no inhibition to $23 \mathrm{~mm}$. In Figure 3, we report the H. pylori growth inhibition caused by Lactobacillus spp.
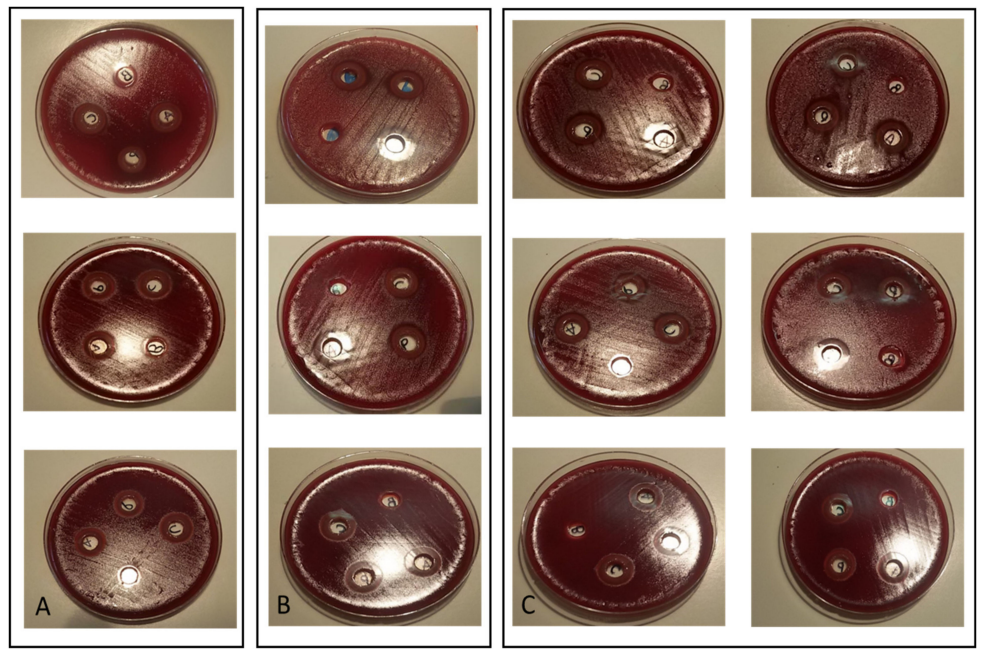

Figure 3. IZs from L. casei, L. paracasei, L. acidophilus (negative control, MRS broth). H. pylori susceptibility test was carried out vs. clarithromycin, metronidazole and levofloxacin. (A) H. pylori strains susceptible to all antibiotics. (B) H. pylori strains resistant to all antibiotics. (C) H. pylori strains resistant to one or two antibiotics. IZs are clearly visible.

A time-kill study was performed on a multi-resistant H. pylori strain. At T0, T3, T6, T24 and T72 h, H. pylori total viable count was performed. Results are reported in Figure 4.

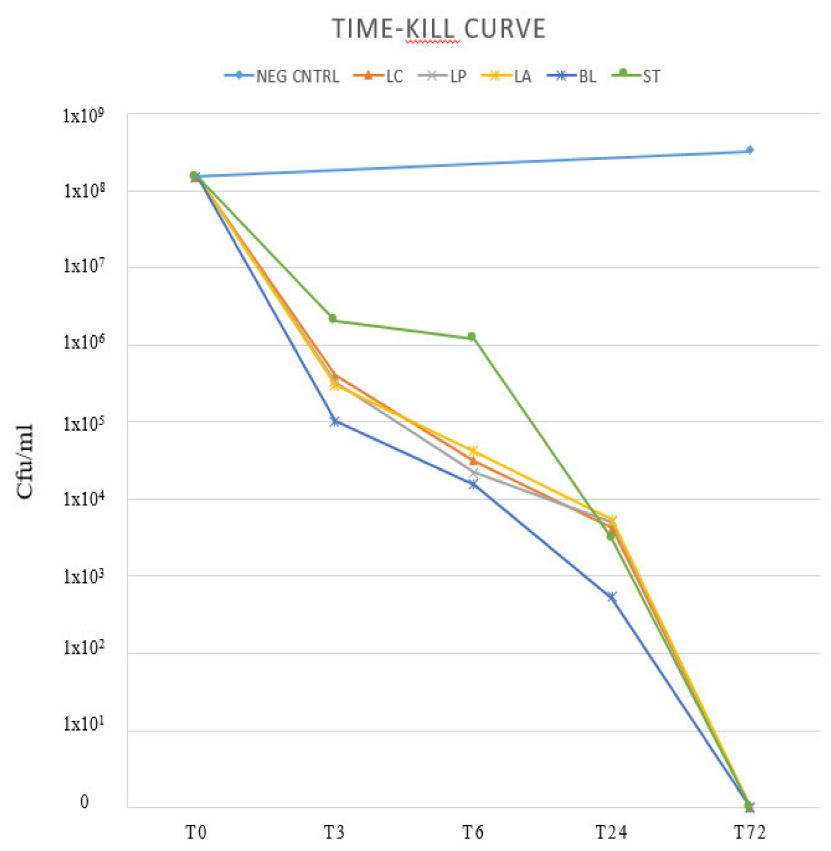

Figure 4. Time-kill curve. One multi-resistant $H$. pylori strain was co-incubated with supernatants derived from overnight broth culture of each probiotic strain. After only $3 \mathrm{~h}$ of incubation, H. pylori viable cell count decreased from $10^{8}$ to $10^{6}-10^{5} \mathrm{CFU} / \mathrm{mL}$ : CFU: colony-forming units. LC: L. casei. LP: $L$. paracasei. LA: L. acidophilus. BL: B. lactis. ST: S. thermophilus. 
At 3 h, H. pylori aliquots co-incubated with L. acidophilus, L. casei, L. paracasei and B. lactis supernatants showed a decrease of $10^{3} \mathrm{CFU} / \mathrm{mL}$ ca. The aliquot co-incubated with $S$. thermophilus supernatant showed a decrease of approximately $10^{2} \mathrm{CFU} / \mathrm{mL}$. The H. pylori viable count continued to decrease steadily until there were no viable cells at $72 \mathrm{~h}$. Viable count of the negative control doubled after $72 \mathrm{~h}$ of incubation.

\section{Discussion}

Research on the gastric microbiota is a recent topic [7], as a consequence of the idea that "the stomach is a sterile organ", inhospitable to bacteria. This short-sighted principle arose because of low gastric $\mathrm{pH}$, duodeno-gastric reflux of bile, thickness of the mucus layer and gastric peristalsis; these are all factors suggesting that bacteria could not exist in such an environment. On the contrary, several Phyla have been identified in the stomach, some present in the gastric lumen (transient colonies) and others colonizing the mucosa (stable colonies). The gastric microbiota is complex and dynamic; it establishes multiple interactions with the mucosa and, when present, also with H. pylori [8-10]. Probiotic bacteria can inhibit $H$. pylori through immunological and/or non-immunological mechanisms. It is known that $H$. pylori infection induces the production of nuclear factor-kB (NF-kB), interleukin-8 (IL-8) and tumor necrosis factor $\alpha(\mathrm{TNF} \alpha)$. IL-8 leads to the migration of neutrophils and monocytes into the mucosa; the activated monocytes and dendritic cells stimulate the production of various cytokines, such as IL-4, IL-5, IL-6 and interferon- $\gamma$ (IFN- $\gamma$ ), leading to an inflammatory reaction (chronic gastritis). The persistence of the inflammatory insult is associated with the development of gastric cancer [11]. Probiotics can modify the immunologic response of the host by interacting with epithelial cells and modulating the secretion of anti-inflammatory cytokines and chemokines, resulting in a reduction of gastric inflammation, mainly through the inhibition of the NF-kB pathway [12,13]. Furthermore, probiotics can enhance the production of secretory IgA, an additional defense against pathogens [14]. Non-immunological mechanisms of probiotics include the production of antimicrobial substances (bacteriocins), inhibition of adherence to the gastric mucosa, stimulation of mucin production and stabilization of the gut mucosal barrier. Furthermore, lactic acid bacteria and bifidobacteria produce organic acids, hydrogen peroxide, carbon dioxide and other antimicrobial compounds that may inhibit potential pathogens. Adhesion of pathogens can be inhibited either by co-aggregation and steric hindrance, or by competing for specific carbohydrate receptors [4,15-17].

H. pylori causes chronic gastritis, peptic ulceration, and may lead to gastric MALT lymphoma and carcinoma development [1]. Treatment of infection remains a challenge for clinicians; in fact, no therapeutic protocol is $100 \%$ effective. What makes the formulation of new therapies fundamental is the substantial increase in drug-resistant strains [2]. In this situation, the role played by the frequent onset of adverse reactions during antibiotic therapies should not be underestimated. They are mainly due to intestinal dysbiosis and consist of the appearance of nausea, vomiting, diarrhea, etc. [3]. These factors notably decrease the compliance of patients to therapy, creating a vicious cycle between adverse reactions, suspension of therapy, clonal selection of resistant bacteria and failure of subsequent therapy. In a recent study, our group observed a drastic increase in resistance rates to all antibiotics tested in patients with at least one failed therapeutic attempt [18]. Given this clear evidence, integration of probiotic strains before and during antibiotic therapy should increase the eradication rates and decrease the onset of dysbiosis-induced adverse events. Although the Maastricht Consensus states that "probiotics associated with antibiotic therapy have positive effects on the management of $H$. pylori infection" [19], meta-analyses of clinical trials published in this field give discrepant results, clearly supporting the need for further research in this area [20-23]. It is not possible to compare published clinical trials because of the extreme variability in terms of probiotic formulations used (type and duration of integration) and antibiotic therapies administered. The same discrepancies can be observed in in vitro studies: some researchers detected different levels of bacteriostatic activity in different probiotic strains [24-27], while others did not detect any inhibition at all [28]. For these reasons, the aim of this study was to assess the in vitro bacteriostatic and bactericidal activity of 
probiotic strains against $H$. pylori, as a preliminary investigation to set up an effective clinical trial. Probiotic strains were selected for their resistance to low $\mathrm{pH}$ and bile salts [29-31] and their growth characteristics. Actinobacteria (Bifidobacterium spp.) create transient colonies, which fluctuate in the gastric lumen and pass into the intestine, while Firmicutes (Lactobacillus spp. and Streptococcus spp.) can form stable colonies in the gastric mucosa [8]. Therefore, we hypothesized that Lactobacillus spp. and S. thermophilus can act directly in the stomach, while B. lactis may work in the intestine, preventing dysbiosis. Furthermore, as noted above, the bactericidal activity of probiotics against $H$. pylori is partially due to bacteriocins. In our opinion, since their identity is not currently fully recognized, it is preferable to use a wide range of bacteriocins, instead of those produced only by one organism, as supplement for $H$. pylori infection treatment. All probiotic strains used showed bacteriostatic and bactericidal activities against H. pylori. Specifically, L. casei, L. paracasei and L. acidophilus showed the best performance since they inhibited $100 \%$ of $H$. pylori strains, regardless of their pattern of antibiotic resistance. S. thermophilus inhibited only $18 \%$ of $H$. pylori strains, but, when present, inhibition halos were among the widest ones (up to $23 \mathrm{~mm}$ ).

Antibiotic resistance mechanisms of $H$. pylori are not fully understood [32]. The known ones should have nothing to do with the mechanisms that make $H$. pylori susceptible to the antimicrobial action of probiotics (at least in vitro, i.e., low $\mathrm{pH}$ and inhibition of urease activity). However, because data on resistance patterns were available, we thought it would be wiser to divide the statistics by "H. pylori resistance patterns", in order to observe if any changes in the efficacy of probiotics were detectable.

Focusing on bactericidal activity, the most interesting information is the drastic decrease of $H$. pylori viable count in the first three hours of incubation: viable cells decreased from $10^{8}$ to $10^{5}$ per $\mathrm{mL}$ $c a$ when co-incubated with Lactobacillus spp. and B. lactis supernatants, and to $10^{6}$ per $\mathrm{mL}$ ca. when co-incubated with $S$. thermophilus supernatants.

It was not possible to normalize the quantity of cells after overnight culture, due to the different bacterial growth rates. Overnight broth-cultures were not diluted in order not to modify their content of metabolites. For homofermentative bacteria, the antimicrobial activity detectable in vitro is mainly due to two factors: the low $\mathrm{pH}$ and lactic acid itself, which may inhibit H. pylori urease enzyme [27]. B. lactis and S. thermophilus growth rates were lower than those of Lactobacillus spp., but, while B. lactis supernatant was as acidic as Lactobacillus spp. supernatant, $S$. thermophilus produced a supernatant with a higher $\mathrm{pH}$. These observations may explain the reason why not all $H$. pylori strains were inhibited by $B$. lactis and $S$. thermophilus in the agar well diffusion test. We can assume that the same two factors (low $\mathrm{pH}$ and lactic acid content) played partial roles in the bactericidal activity assay; in fact, S. thermophilus bactericidal activity was the least effective against $H$. pylori in the first $3 \mathrm{~h}$ of co-incubation.

\section{Materials and Methods}

\subsection{Helicobacter Pylori Culture and Susceptibility Test}

Fifty-seven H. pylori clinical isolates were cultured on the commercial selective medium Pylori Agar (BioMérieux S.p.A. Florence, Italy). The plates were incubated in jars under microaerobic conditions (CampyGen GasPack, Oxoid S.p.A Milan, Italy) at $37^{\circ} \mathrm{C}$ for 3 to 5 days. The colonies resembling $H$. pylori were identified by Gram stain and oxidase, catalase and urease tests. $H$. pylori colonies were then suspended in sterile saline solution at a density corresponding to McFarland opacity standard \#4 ( $1 \mathrm{McF}=3 \times 10^{8}$ cells $\left./ \mathrm{mL}\right)$ to perform antibiotic susceptibility test vs. clarithromycin, metronidazole and levofloxacin. The E-Test method was used as follows: a total of 4 agar plates for every $H$. pylori strain were streaked in 3 directions with a swab dipped into each bacterial suspension to produce a lawn of growth. Three E-Test strips (BioMérieux S.p.A. Florence, Italy) were placed onto three plates (one strip per plate), which were incubated immediately in a microaerobic atmosphere at $37^{\circ} \mathrm{C}$ for $72 \mathrm{~h}$. A fourth plate was used as positive control of bacterial development. Clarithromycin, metronidazole and levofloxacin resistance break points for the minimal inhibitory concentration (MIC) 
were: greater than $0.5 \mathrm{mg} / \mathrm{L}$, greater than $8 \mathrm{mg} / \mathrm{L}$ and greater than $1 \mathrm{mg} / \mathrm{L}$, respectively, according to the updated recommendations of the European Committee on Antimicrobial Susceptibility Testing (EUCAST 2020) [33]. All tests were carried out in duplicate.

\subsection{Probiotic Strains}

Lactobacillus casei DGDG (Sofar S.p.A., Milan, Italy), Lactobacillus paracasei LPC-S01 (Sofar S.p.A., Milan, Italy), Lactobacillus acidophilus LA14 (Danisco S.p.A. Milan, Italy), Bifidobacterium lactis BL04 (Danisco S.p.A. Milan, Italy), Streptococcus thermophilus ST21 (Danisco S.p.A., Milan, Italy) were tested. Lyophilized L. casei, L. paracasei, L. acidophilus and B. lactis were suspended in saline solution and seeded on DeMan-Rogosa-Sharpe (MRS) agar (Oxoid S.p.A.,Milan, Italy) with $0.05 \%$ L-cysteine, incubated in jar for $24 \mathrm{~h}$ at $37^{\circ} \mathrm{C}$ in an anaerobic atmosphere (AnaeroGen GasPack, Oxoid S.p.A., Milan, Italy). Lyophilized $S$. thermophilus was suspended in saline solution and seeded onto blood agar containing $5 \%$ horse blood (HB) (Kima S.r.L., Padova, Italy), incubated in jars for $24 \mathrm{~h}$ at $37^{\circ} \mathrm{C}$ under microaerobic conditions (CO2Gen GasPack, Oxoid S.p.A., Milan, Italy). To confirm bacterial species identification, matrix-assisted laser desorption ionization-time of flight mass spectrometry (Maldi-Tof microflex, Bruker Daltonics S.R.L., Macerata, Italy - SciLsLabSoftware, 3D version 2016b (Bruker Daltonics S.r.L, Macerata, Italy) was performed [34].

\subsection{Overnight Broth Cultures}

Two colonies of L. casei, L. paracasei, L. acidophilus and B. lactis were suspended in $10 \mathrm{~mL}$ of MRS broth. Two colonies of $S$. thermophilus were suspended in $10 \mathrm{~mL}$ of brain heart infusion (BHI) broth (Oxoid S.p.A., Milan, Italy). Suspensions were kept for $20 \mathrm{~h}$ under specific culture conditions (24 h at $37^{\circ} \mathrm{C}$ in anaerobiosis for Lactobacillus spp. and B. lactis, $24 \mathrm{~h}$ at $37^{\circ} \mathrm{C}$ in $\mathrm{CO}_{2}$ enriched atmosphere for S. thermophilus).

\subsection{Supernatants}

Overnight cultures were centrifuged at $2500 \times g$ for $15 \mathrm{~min}$ and supernatants were filtered through $0.22 \mu \mathrm{m}$ pore size membranes.

\subsection{Agar Well Diffusion (Bacteriostatic Activity).}

Fifty-seven clinical $H$. pylori isolates were used. Each strain was suspended in saline solution at a density corresponding to McFarland opacity standard \#2 and seeded onto Mueller-Hinto fastidious (MHF) agar (Kima S.r.L, Padova, Italy) using a sterile swab. With the aid of a sterile tip, 7 wells (7 mm diameter) were drilled into the agar. Then, $100 \mu \mathrm{L}$ of each overnight culture was deposited into 5 wells and $100 \mu \mathrm{L}$ of plane broth was deposited into 2 wells for negative control. Growth inhibition zones (IZs) were read after $72 \mathrm{~h}$ of incubation at $37^{\circ} \mathrm{C}$ in microaerobic environment. IZs were reported as the diameters of growth inhibition (well diameter was subtracted). Agar well diffusion method was carried out following the scheme reported in Figure 5.

\subsection{Time-Kill Curve (Bactericidal Activity)}

One H. pylori strain resistant to clarithromycin, metronidazole and levofloxacin was co-incubated with the supernatant (derived from overnight culture) of each one of the five probiotic strains. The $H$. pylori strain was suspended in modified Brucella broth (MBB) at a density corresponding to McFarland opacity standard \#1, then the suspension was divided in 6 aliquots. A total of 5 were co-incubated with probiotic supernatants $(1: 2 v / v)$; the sixth aliquot was diluted 1:2 with MBB and was used as a negative control. The mixtures were thereafter incubated at $37^{\circ} \mathrm{C}$ in jars under microaerobic conditions (jars were placed on a stirrer). At T0-3-6-24-72 h, scalar dilutions (1:10) were performed to determine the viable count. From each dilution, $100 \mu \mathrm{L}$ was subcultured onto agar plates that were incubated in microaerobiosis at $37^{\circ} \mathrm{C}$ for 3-5 days. Plates were then inspected and the colonies were counted; 
their number was expressed as colony forming unit (CFU) per $\mathrm{mL}$. A time-kill curve was carried out following the scheme reported in Figure 6. All tests were performed in duplicate.

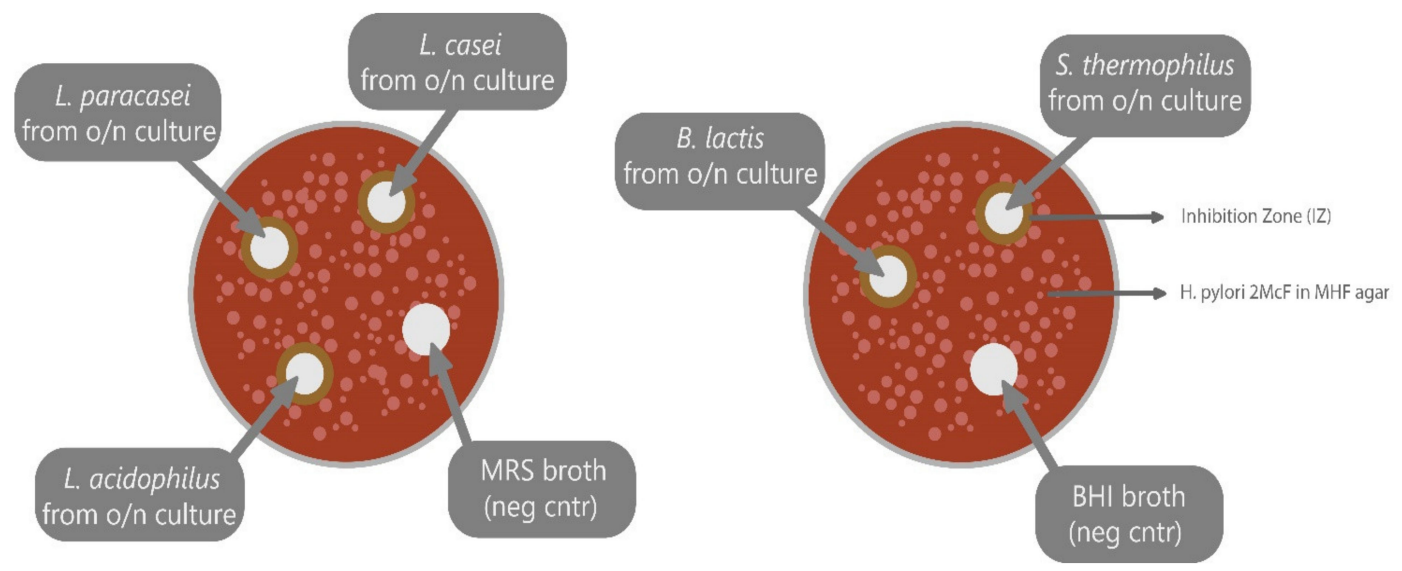

Figure 5. Agar well diffusion seeding scheme. o/n: overnight. McF: McFarland. MHF: Mueller-Hinton-Fastidious agar.

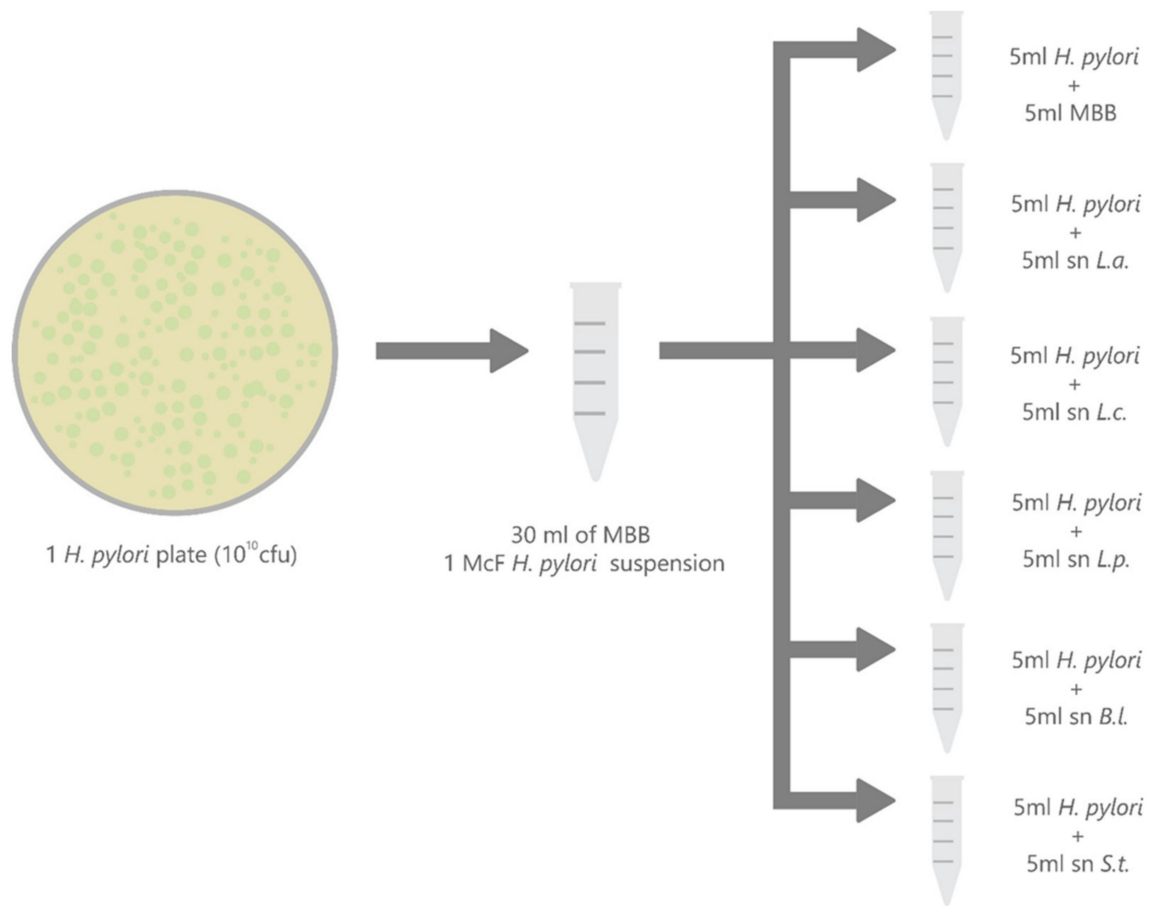

Figure 6. Aliquots of $H$. pylori suspension co-incubated with supernatants (sn) of probiotic strains $(1: 2 v / v)$. CFU: colony forming unit. MBB: modified brucella broth. L.a.: L. acidophilus. L.c.: L. casei. L.p.: l. paracasei. B.1.: B. lactis. S.t.: S. thermophilus.

\subsection{Statistical Analysis}

Student's $t$-test and ANOVA were used for comparison between independent samples. A $p$ value less than 0.05 was considered significant. Statistical analysis was performed with MedCalc19.1.

\section{Conclusions}

All five considered probiotic strains showed both bacteriostatic and bactericidal activity against $H$. pylori in vitro. L. casei, L. paracasei and L. acidophilus were the most effective in both tests. These results must be validated in vivo by a randomized clinical trial, which will enable us to assess the putative 
increase of eradication rates and the potential decrease of adverse events. The correct use of probiotics as adjuvants in antibiotic therapy against $H$. pylori could represent a turning point in the management of $H$. pylori positive patients, especially in cases of multidrug resistance.

Author Contributions: Guarantor of the article: B.V., J.H., T.L. and N.F. conceived the study. I.M.S., V.P., C.F., G.P. and M.P. performed the microbiological analyses. I.M.S., M.P. and L.S. performed data management and statistical analysis. I.M.S., M.P. and L.S. wrote the manuscript. G.F., G.B., and C.B. critically reviewed the manuscript. All authors have read and agreed to the published version of the manuscript.

Funding: This research received no external funding.

Acknowledgments: The authors received no financial support for the research or publication of this article.

Conflicts of Interest: All authors have no conflict of interest to disclose.

\section{References}

1. Holton, J.; Figura, N.; Vaira, D. Helicobacter pylori: Atlas of Investigation and Management; Clinical Publishing: Oxford, UK, 2012.

2. Saracino, I.M.; Fiorini, G.; Zullo, A.; Pavoni, M.; Saccomanno, L.; Vaira, D. Trends in primary antibiotic resistance in H.pylori strains isolated in Italy between 2009 and 2019. Antibiotics 2020, 9, 26. [CrossRef]

3. Ruggiero, P. Use of probiotics in the fight against Helicobacter pylori. World J. Gastrointest. Pathophys. 2014, 5, 384-391. [CrossRef] [PubMed]

4. Patel, A.; Shah, N.; Prajapati, J.B. Clinical application of probiotics in the treatment of Helicobacter pylori infection. A brief review. J. Microbiol. Immunol. Infect. 2014, 47, 429-437. [CrossRef] [PubMed]

5. Bathia, S.J.; Kochar, N.; Abraham, P.; Nair, N.G.; Metha, A.P. Lactobacillus acidophilus Inhibits Growth of Campylobacter pylori In Vitro. J. Clin. Microbiol. 1989, 27, 2328-2330.

6. Shi, X.; Zhang, J.; Mo, L.; Shi, J.; Qin, M.; Huang, X. Efficacy and safety of probiotics in eradicating Helicobacter pylori A network meta-analysis. Medicine 2019, 98, 7.

7. Fuhler, G.M.; Hecht, G.A.; Foley, S.E.; Lebron, A.C.; Hazleton, K. 2019 Gut Microbiota for Health World Summit. Gastroenterology 2020, 158, 28-31. [CrossRef]

8. Nardone, G.; Compare, D. The human gastric microbiota: Is it time to rethink the pathogenesis of stomach diseases? UEG J. 2015, 3, 255-260. [CrossRef]

9. Brawner, K.M.; Morrow, C.D.; Smith, P.D. Gastric Microbiome and Gastric Cancer. Cancer J. 2014, 3, 211-216. [CrossRef]

10. Cao, L.; Yu, J. Effect of Helicobacter pylori Infection on the Composition of Gastric Microbiota in the Development of Gastric Cancer. Gastrointest. Tumors 2015, 2, 14-25. [CrossRef]

11. Perdigon, G.; Medina, M.; Vintiñi, E.; Valdéz, J.C. Intestinal pathway of internalization of lactic acid bacteria and gut mucosal immunostimulation. Int. J. Immunopathol. Pharmacol. 2000, 13, 141-150.

12. Yang, Y.J.; Chuang, C.C.; Yang, H.B.; Lu, C.C.; Sheu, B.S. Lactobacillus acidophilus ameliorates, H. pylori induced gastric inflammation by inactivating the Smad7 and NFkB pathways. BMC Microbiol. 2012, 12, 1-8. [CrossRef] [PubMed]

13. Murosaki, S.; Muroyama, K.; Yamamoto, Y.; Yoshikai, Y. Antitumor effect of heat-killed Lactobacillus plantarum L-137 through restoration of impaired interleukin-12 production in tumorbearing mice. Cancer Immunol. Immunother. 2000, 49, 157-164. [CrossRef] [PubMed]

14. Kabir, A.M.; Aiba, Y.; Takagi, A.; Kamiya, S.; Miwa, T.; Koga, Y. Prevention of Helicobacter pylori infection by lactobacilli in a gnotobiotic murine model. Gut 1997, 41, 49-55. [CrossRef] [PubMed]

15. Mack, D.R.; Ahrne, S.; Hyde, L.; Wei, S.; Hollingsworth, M.A. Extracellular MUC3 mucin secretion follows adherence of Lactobacillus strains to intestinal epithelial cells in vitro. Gut 2003, 52, 827-833. [CrossRef]

16. Mattar, A.F.; Teitelbaum, D.H.; Drongowski, R.A.; Yongyi, F.; Harmon, C.M.; Coran, A.G. Probiotics up-regulate MUC-2 mucin gene expression in a Caco-2 cell-culture model. Pediatr. Surg. Int. 2002, 18, 586-590. [PubMed]

17. Sanz, Y. Antimicrobial peptides are among the antagonistic metabolites produced by Bifidobacterium against Helicobacter pylori. Int. J. Antimicrob. Agents 2005, 25, 385-389. 
18. Saracino, I.M.; Pavoni, M.; Zullo, A.; Fiorini, G.; Saccomanno, L.; Lazzarotto, T.; Cavallo, R.; Antonelli, G.; Vaira, D. Antibiotic resistance and therapy outcome in H.pylori eradication failure patients. Antiobiotics 2020, $9,121$.

19. Malfertheiner, P.; Megraud,F.; O’Morain, C.A.; Atherton, J.; Axon, A.T.; Bazzoli, F.; El-Omar, E.M. Management of Helicobacter pylori Infection-The Maastricht IV/Florence Consensus Report. Gut 2012, 61, 646-664. [CrossRef]

20. Wang, Z.-H.; Gao, Q.-Y.; Fang, J.-Y. Meta-Analysis of the Efficacy and Safety of Lactobacillus-containing and Bifidobacterium-containing Probiotic Compound Preparation in Helicobacter pylori Eradication Therapy. J. Clin. Gastroenterol. 2013, 47, 25-32. [CrossRef]

21. Tomás, N.-R.; Fernando, M.S.; Ricardo, C.B.; Rejane, M.J.; Prado, M.-F.; de Oliveira, M.N.; Bogsan, C.S.; Chinzon, D.; Eisig, J.N. Association of a probiotic to a Helicobacter pylori eradication regimen does not increase efficacy or decreases the adverse effects of the treatment: A prospective, randomized, double-blind, placebo-controlled study. BMC Gastroenterol. 2013, 13, 56.

22. Lau, C.S.M.; Ward, A.; Chamberlain, R.S. Probiotics improve the efficacy of standard triple therapy in the eradication of Helicobacter pylori: A meta-analysis. Infect. Drug Resist. 2016, 9, 275-289. [CrossRef] [PubMed]

23. Shakya, S.; Zhang, G.Q.; Hu, H.J.; Li, Z.Y. Lactobacillus GG Supplementation on Anti-Helicobacter pylori Therapy-Related Side Effects and Eradication Rates: A Meta-Analysis. Open J. Internal Med. 2015, 5, 96-105. [CrossRef]

24. Enany, S.; Abdalla, S. In vitro antagonistic activity of Lactobacillus casei against Helicobacter pylori. Brazil. J. Microbiol. 2015, 46, 1201-1206. [CrossRef] [PubMed]

25. Boyanova, L.; Stephanova-Kondratenko, M.; Mitov, I. Anti-Helicobacter pylori activity of Lactobacillus delbrueckii subsp. bulgaricus strains: Preliminary report. Soc. Appl. Microbiol. Lett. Appl. Microbiol. 2009, 48, 579-584. [CrossRef] [PubMed]

26. Medouakh, A.; Abdeslam, A.; Belsotane, A. Antagonistic activity of Lactobacillus sp. against Helicobacter pylori. Int. J. Microbiol. Res. 2010, 1, 80-86.

27. Sgouras, D.; Maragkoudakis, P.; Petraki, K.; Martinez-Gonzalez, B.; Eriotou, E.; Michopoulos, S.; Kalantzopoulos, G.; Tsakalidou, A.; Mentis, E. In Vitro and In Vivo Inhibition of Helicobacter pylori by Lactobacillus casei Strain Shirota. Appl. Environ. Microbiol. 2004, 70, 518-526. [CrossRef] [PubMed]

28. Cats, A.; Kuipers, E.J.; Bosschaert, M.A.; Pot, R.G.J.; Vandenbrouckergrauls, C.M.J.E.; Kusters, J.G. Effect of frequent consumption of a Lactobacillus casei-containing milk drink in Helicobacter pylori-colonized subjects. Aliment. Pharmacol. Ther. 2003, 17, 429-435. [CrossRef]

29. Boke, H.; Aslim, B.; Alp, G. Rore of resistance to bile salts and acid tolerance of exopolysaccarides (EPSS) produced by yogurt starter bacteria. Arch. Biol. Sci. Belgrade 2014, 62, 323-328. [CrossRef]

30. Ruiz, L.; Margolles, A.; Sánchez, B. Bile resistance mechanisms in Lactobacillus and Bifidobacterium. Front. Microbial. 2013, 4, 1-8.

31. Fuochi, V.; Petronio, G.; Lissandrello, E.; Furneri, P.M. Evaluation of resistance to low pH and bile salts of human Lactobacillus spp. Isolates. Int. J. Immunopathol. Pharmacol. 2015, 28, 426-433. [CrossRef]

32. De Francesco, V.; Zullo, A.; Hassan, C.; Giorgio, F.; Rosania, R.; Ierardi, E. Mechanisms of Helicobacter pylori antibiotic resistance: An updated appraisal. World J. Gastrointest. Pathophysiol. 2011, 2, 35-41. [CrossRef] [PubMed]

33. Clinical Breakpoints-Breakpoints and Guidance. Available online: http://www.eucast.org/clinical_ breakpoints (accessed on 7 January 2020).

34. Murray, P.R. What Is New in Clinical Microbiology-Microbial Identification by MALDI-TOF Mass Spectrometry. J. Mol. Diagn. 2012, 14, 419-423. [CrossRef] [PubMed]

(C) 2020 by the authors. Licensee MDPI, Basel, Switzerland. This article is an open access article distributed under the terms and conditions of the Creative Commons Attribution (CC BY) license (http://creativecommons.org/licenses/by/4.0/). 